\title{
Real-Time Incident Detection and Capacity Estimation Using Loop Detector Data
}

\author{
Syed Muzammil Abbas Rizvi, ${ }^{1}$ Afzal Ahmed $\mathbb{D},{ }^{2}$ and Yongjun Shen $\mathbb{D}^{1}$ \\ ${ }^{1}$ Southeast University, Nanjing, China \\ ${ }^{2}$ NED University of Engineering and Technology, Karachi, Pakistan \\ Correspondence should be addressed to Yongjun Shen; shenyongjun@seu.edu.cn
}

Received 21 June 2020; Revised 16 August 2020; Accepted 15 September 2020; Published 7 October 2020

Academic Editor: Giuseppe Guido

Copyright (C) 2020 Syed Muzammil Abbas Rizvi et al. This is an open access article distributed under the Creative Commons Attribution License, which permits unrestricted use, distribution, and reproduction in any medium, provided the original work is properly cited.

\begin{abstract}
Given the fact that the existing literature lacks the real-time estimation of road capacity and incident location using data from inductance loop detectors, a data-driven framework is proposed in this study for real-time incident detection, as well as road capacity and incident location estimation. The proposed algorithm for incident detection is developed based on the variation in traffic flow parameters acquired from inductance loop detectors. Threshold values of speed and occupancy are determined for incident detection based on the PeMS database. The detection of the incident is followed by the real-time road capacity and incident location estimation using a Cell Transmission Model (CTM) based approach. The data of several incidents were downloaded from PeMS and used for the development of the proposed framework presented in this study. Results show that the developed framework detects the incident and estimates the reduced capacity accurately. The location of the incident is estimated with an overall accuracy of $92.5 \%$. The performance of the proposed framework can be further improved by incorporating the effect of the on-ramps, off-ramps, and high-occupancy lanes, as well as by modeling each lane separately.
\end{abstract}

\section{Introduction}

An incident is among the most unfavorable events that significantly affect the roadway capacity and decrease the reliability of the system. The impact of disruption due to incident could be minimized by implementing real-time intervention strategies. The effectiveness of the intervention measures depends on accurate real-time information about the location, duration, and impact of the incident.

The main problem caused by the incident is not the lack of capacity but the temporary reduction of capacity due to the incident [1]. Golob et al. [2] found a strong relationship between the likelihood of incidents and traffic conditions. Sun and $\mathrm{Li}$ [3] investigated the relationship between incidents and different traffic conditions. Mattsson and Jenelius [4] calculated the increment in the travel time of links when certain links in the network are closed. Goldberg [5] found that, with an increase in capacity utilization, the redundancy of the system decreases, making the system more vulnerable.
Among traffic flow parameters, the capacity of the road section is directly affected by the incident, which then affects the other parameters such as speed, delay, and occupancy.

The capacity of the road is not a fixed value. It varies with the change in traffic conditions, road environment, and weather conditions. Estimating the capacity round the clock is a cumbersome task. The capacity estimation becomes more critical when an incident occurs, as a significant part of the delay faced by drivers is due to the incident $[6,7]$. Ahmed and Hawas [8] introduced a threshold-based approach for incident detection and validated the model through simulation data. Sheu [9] used traffic flow and occupancy data of I-880 freeway in Oakland, California, for real-time incident detection using discrete-time, nonlinear, stochastic system modeling. Rossi and Gastaldi [10] used fuzzy logic for incident detection from loop detector data. Zhang and He [11] and $\mathrm{Gu}$ and Qian [12] developed a deep learning approach for detecting the incident through social media. Xiao [13] proposed a model based on ensemble learning for incident 
detection. Kinoshita and Takasu [14] formulated a simple model for incident detection from probe car data. Oh [15] conducted statistical analyses and identified traffic conditions that lead to traffic incident likelihood. Chen et al. [16] determined factors which contribute to increasing the likelihood of incidents on I-25. Wang and Papageorgiou [17] estimated the real-time traffic state using the loop detector and predicted the traffic state by a second-order traffic flow model. Ngoduy [18] developed a technique for estimating the real-time traffic state for a section of freeway using a particle filtering algorithm. CTM-EKF (Cell Transmission Model-Extended Kalman Filter-) based technique has been applied for real-time traffic state estimation for traffic network disrupted with an incident [19-21].

Real-time capacity estimation is beneficial when there is a significant reduction in capacity due to events such as incidents. Chen et al. [22] proposed a zero-inflated negative binomial (ZINB) model for incident risk information, which can be beneficial for traffic management. Traffic incident is a random event, and reduced capacity can be modeled as a random variable [23]. Hadi and Sinha [24] used multiple pieces of software and estimated the incident capacity at different trials. Knoop and Hoogendoorn [6] collected data from a helicopter and estimated incident capacity through microsimulation. The reduced capacity due to the incident creates congestion for the upstream traffic. If the information is not disseminated to the drivers at the upstream of the incident location, then congestion will prevail, and longer queues will be formed. The increased upstream occupancy is associated with more severe incidents [25]. Smith and Qin [23] analyzed a large dataset of traffic flow and incident data and found a $63 \%$ reduction in capacity when one lane of traffic was blocked, while $77 \%$ reduction was observed when two of three lanes were blocked. Almotahari and Yazici [26] used PeMS database for estimation of delay caused by reduced capacity after the incident. Bertini and Myton [27] identified bottlenecks on the freeways in California using PeMS database.

The estimation of the location of an incident enables the traffic control system to divert the flow to an alternate route to reduce congestion on the affected road. In the literature, the offline estimation of the incident location has been performed using incident datasets. One or multiple locations were identified, which were vulnerable to the incident. These locations are called blackspots or hotspots, having a high probability of an incident. Blackspot analysis requires spatial and temporal data for determining the precise location [28-31]. The location of an incident can be identified by kernel density and K-means clustering in GIS to estimate the locations with a high probability of an incident [32-35]. The location of the incident in the existing literature is mostly estimated offline, and a real-time incident location estimation framework is lacking.

The impact of capacity reduction due to incidents can be minimized by implementing real-time optimal intervention strategies. The change in information of traffic flow parameters due to disruption events can be used to prompt the request for initiating the intervention. The information about capacity variation helps traffic management system to manage the traffic optimally and disseminate correct information to drivers. Intelligent Transportation System (ITS) based applications for real-time dynamic traffic assignments such as ATIS (Advanced Traveller Information Systems) and ATCS (Adaptive Traffic Control Systems) can be made more accurate and useful using reliable information about the impact, time, and location of the incident. The accuracy of the traffic state estimation depends on the location of the incident with respect to the sensor [36]. The time of road-crash is also essential to divert the traffic to alternative routes. Diversion of traffic is implemented until the capacity of the road is restored to its normal value. The suboptimal diversion strategy may lead to increased congestion on the road network and intensify the impact of the incident [37].

The review of the literature indicates that a significant amount of research has been done in the domain of capacity estimation. Previous studies, along with other strategies, considered the capacity as a dynamic parameter. However, an explicit framework for real-time road capacity and incident location estimation using loop detector data does not exist in the available literature. This study develops a framework for incident detection, capacity estimation, and estimation of incident location based on data of traffic flow parameters obtained from loop detectors. The proposed framework is based on the open-source data of the California State Department of Transportation (PeMS). Incident data, along with traffic flow data, are analyzed to develop the proposed framework. The change in traffic flow parameters and congestion developed upstream after the incident will be used to detect the incident and estimation of the incident location, respectively.

\section{Model Development}

Real-time estimation algorithms need to collect, process, and update the data to the system in a very short interval of time, which can be achieved by minimizing the number of variables and developing computationally efficient algorithms. The macroscopic traffic flow parameters provide essential information that is enough for the estimation of the traffic state. Loop detectors measure macroscopic traffic data of flow, speed, and occupancy in real-time at fixed time intervals. These loop detectors are embedded in the pavement and provide continuous temporal information for that point. The spatial information between the detectors can be captured by using traffic flow models such as the Cell Transmission Model (CTM) with measurements acquired from the sensors.

The traffic flow parameters measured at the loop detectors are affected by the incident due to the reduction of capacity. The variation of traffic flow parameters due to the incident can be used for incident detection, estimation of capacity, and estimating the location of the incident in a road section between two consecutive loop detectors. However, variation in the traffic flow parameters recorded at the sensor could be due to the factors other than the incident such as the daily variation, weekly variation, or variation due to the weather impact. Therefore, an appropriate incident 


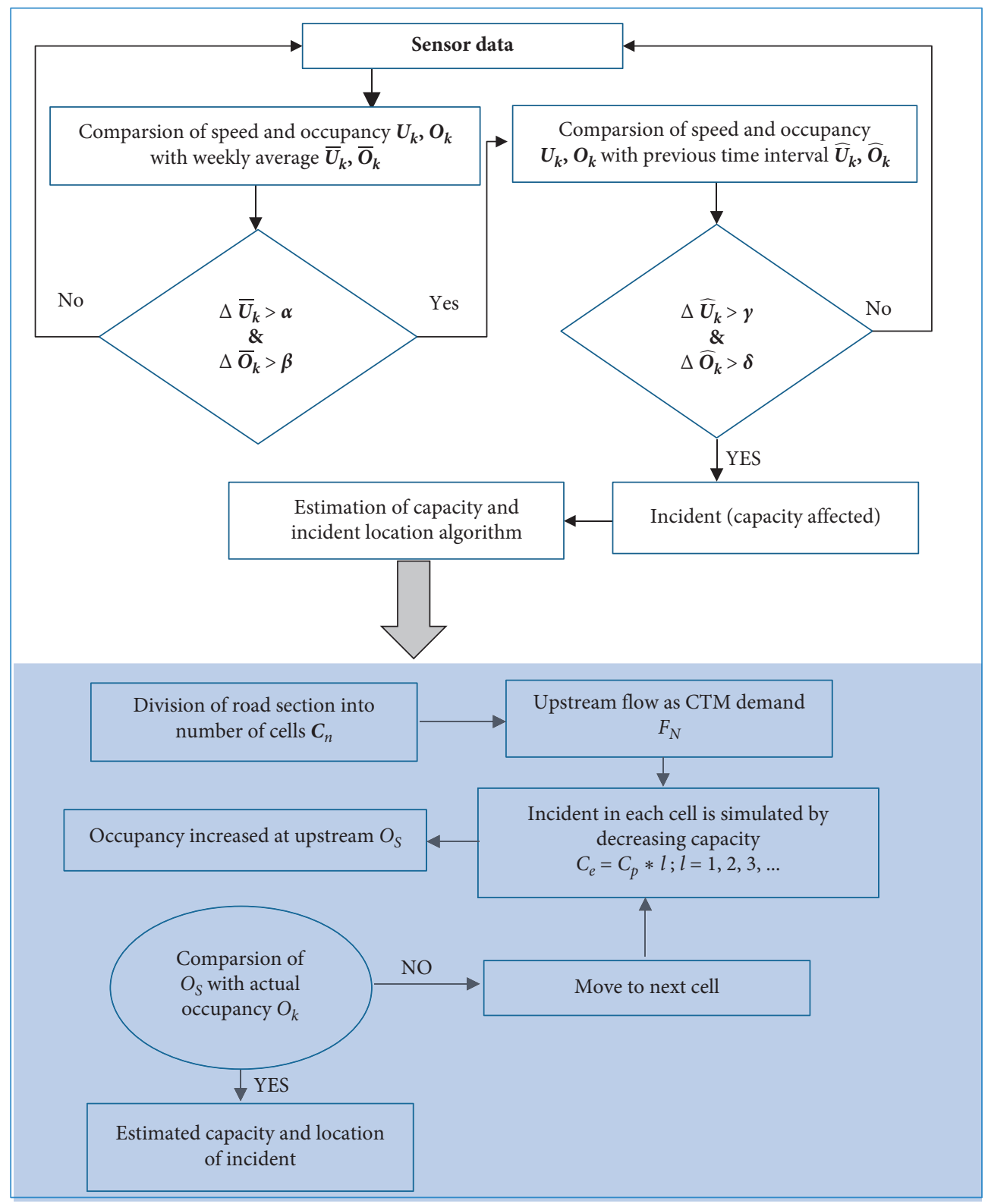

FIgURE 1: Overall framework of this research.

detection model is needed to detect false alarms and improve the efficiency of the incident detection process.

In this study, the proposed algorithm continuously evaluates the information acquired from sensors to detect the incident. The detection of incident triggers the algorithm for capacity and the location of the incident estimation. The incident is detected when variation in traffic flow parameters like speed and occupancy exceeds predefined threshold limits. The threshold limits are established by performing a comparative analysis of post-incident data with the data during normal traffic conditions. If the difference of speed $\triangle \bar{U}_{k}$ and the difference in occupancy $\triangle \bar{O}_{k}$ at current time $k$ with the weekly average are higher than the threshold limits $\alpha$ and $\beta$, respectively, then there might be a possibility of an incident. This is verified by further comparing the current speed and occupancy values with the average values of these parameters for the past 15 minutes ( $k: 15 \mathrm{~min})$. The incident is detected when the difference of speed and occupancy with previous three-time intervals $\triangle \widehat{U}_{k}, \triangle \widehat{O}_{k}$ also exceeds the threshold limits $\gamma$ and $\delta$, respectively. Figure 1 describes the overall framework for this research, and the list of variables with their symbols is shown in Figure 2.

Various components of the overall research framework are described in the following subsections.

2.1. Incident Detection. The PeMS database contains detailed information on incidents, including spatiotemporal information like time, date, day, location, and duration. Moreover, it also contains the type of incident and incident severity. The incident causes congestion at the upstream sensor when traffic demand is higher, and the impact of the 


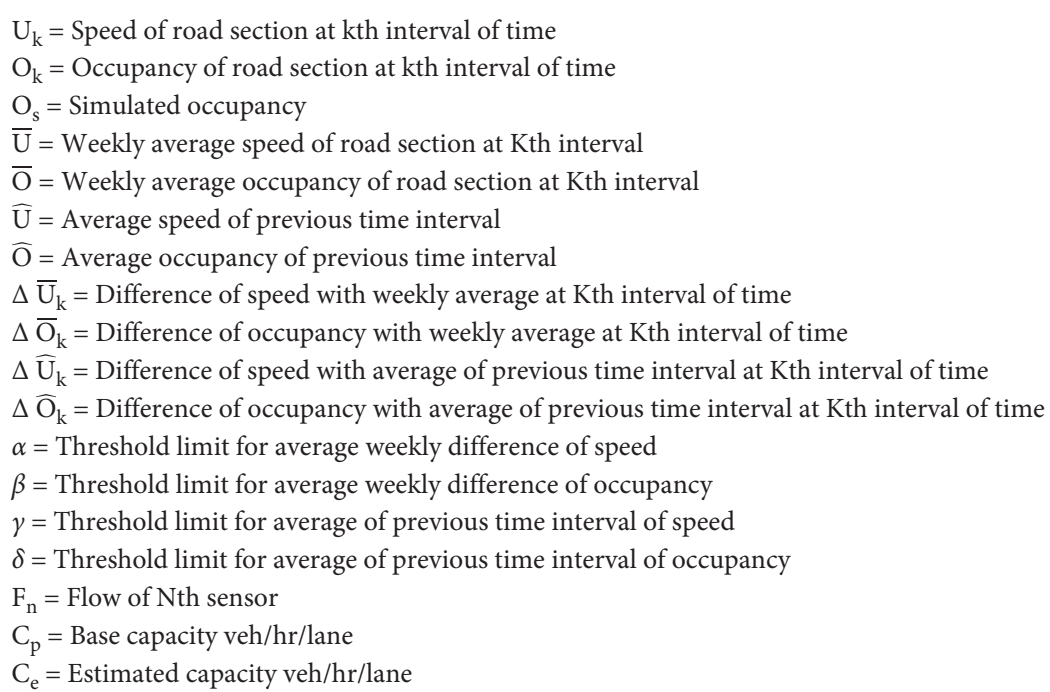

FIgURE 2: List of variables with their symbols.

accident is also significant to cause congestion. The incidents occurring when traffic demand is low, or when the impact of the accident is significantly low to cause any congestion, are not included in the scope of this study. Furthermore, modeling such incidents is not significant in devising realtime traffic control and incident management strategies. Therefore, in this study, only those incidents are selected, which resulted in oversaturated flow conditions as the proposed framework relies on the sensor information.

The data of numerous accidents were downloaded, out of which nine incident scenarios are analyzed. Traffic data are collected from the upstream sensor of incident location reported in the incident report of the PeMS database. For incident detection, the data of four weeks before the incident date were collected to determine the average weekly values of occupancy and speed.

Figure 3 shows the change in speed recorded at the upstream sensor of the incident location and compares it with the average weekly speed, which is estimated by taking the average of speeds observed on the same day for the past four weeks. The speed of vehicles significantly reduced after the incident, and the comparison of speed with the weekly average shows an evident trend that can be used to detect the incident. However, the detection of the incident cannot rely only on one criterion. Therefore, the comparison of occupancy with the weekly average was also included in the incident detection model. Figure 4 shows the change in occupancy measured at the upstream sensor during the incident. The occupancy at the upstream sensor was significantly increased after the incident, which is used as one of the criteria for incident detection.

The time of the incident from PeMS data of the selected freeway sections is shown in Table 1, along with measured speed and occupancy after the incident. The data provided in PeMS database aggregate speed and occupancy for 5-minute intervals. Due to the 5-minute aggregation, the effect of the incident on speed and occupancy is diluted, and, in some cases, it takes 1-2 intervals to show the impact of the incident on the upstream sensor data. This lag can also be attributed to the time taken in the propagation of traffic congestion towards the upstream sensor after the incident.

The proposed incident detection model detects the incident based on the difference in four parameters. These four parameters are based on the difference in current speed and occupancy with their corresponding weekly average and the average of the previous 15 minutes (three intervals). The threshold values of these parameters can be derived based on the data presented in Table 2. The higher threshold values of these parameters will reduce false detection. However, there could be a possibility of missing the accident with too high threshold values. Similarly, the lower threshold values will ensure the maximum possibility of incident detection and could also result in some false detections.

The optimum threshold values were estimated by modeling all the incident locations using the four abovementioned parameters by changing their values from $10 \%$ to $90 \%$ with an interval of $10 \%$. The maximum difference in parameter values that detected all the incidents was selected as the threshold value for that parameter. Table 3 shows the number of incidents detected with different values of the parameters and highlights the cells corresponding to the threshold values.

Based on Table 3, the threshold values of the parameters are taken as follows:

$$
\begin{aligned}
& \boldsymbol{\alpha}=30, \\
& \boldsymbol{\beta}=10, \\
& \gamma=10, \\
& \boldsymbol{\delta}=30 .
\end{aligned}
$$

2.2. Estimation of Road Capacity and Incident Location. The proposed algorithm for estimation of road capacity and incident location uses the Cell Transmission Model (CTM) [38], which divides a road segment into 

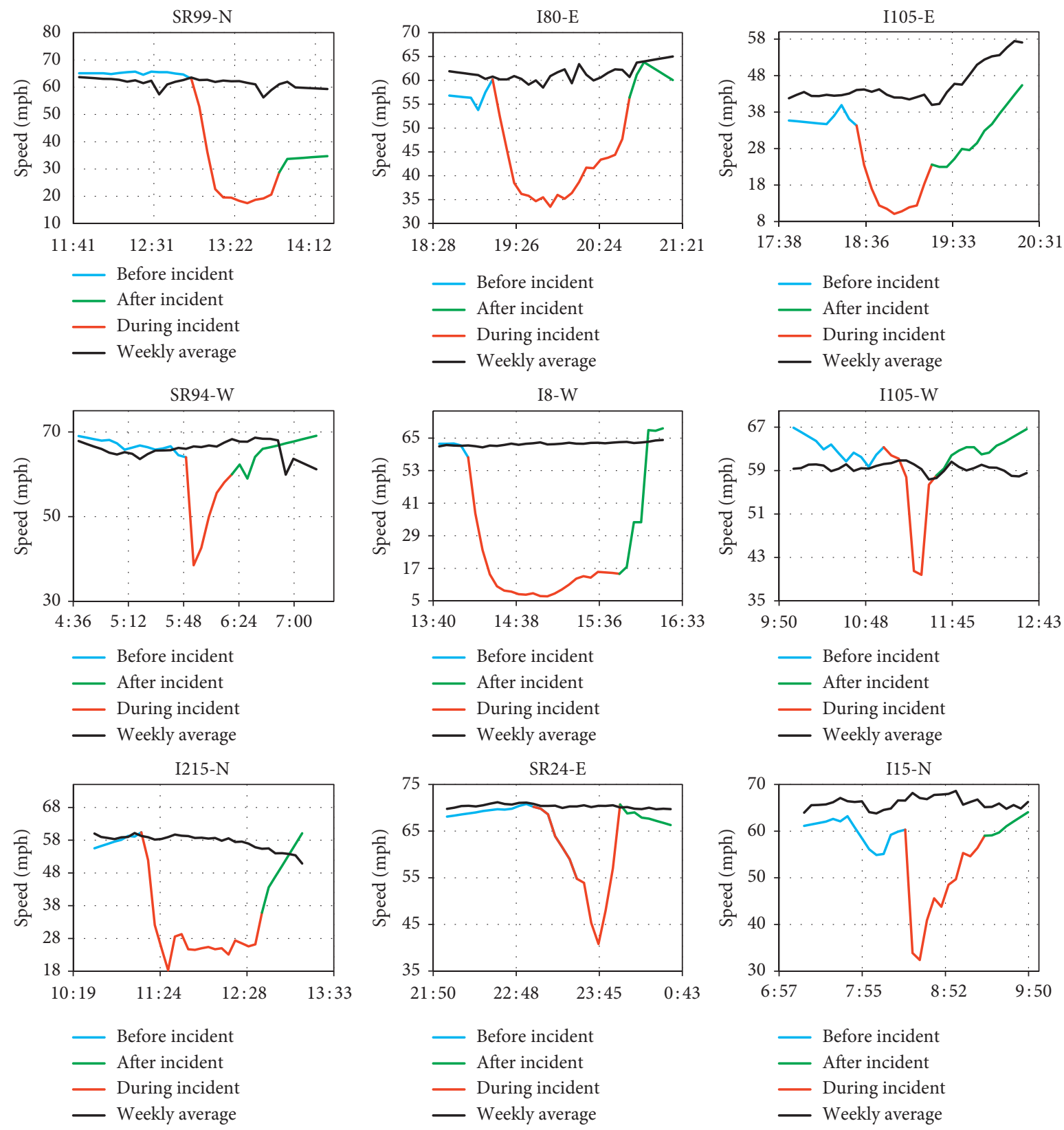

FIGURE 3: The comparison of pre-incident, during the incident and post-incident speeds with the weekly average speed.

homogenous cells of equal lengths. In equation (2), the CTM updates traffic occupancy $n_{i}$ for each future timestep $k+1$ and all the cells in a road network. $y_{i}$ is the outflow from cell $i$.

$$
\begin{aligned}
n_{i}(k+1) & =n_{i}(k)+y_{i}(k)-y_{i+1}(k), \\
y_{i}(k) & =\min \left(n_{i-1}(k), Q_{i}(k), N_{i}(k)-n_{i}(k)\right) .
\end{aligned}
$$

In equation (3), $Q_{i}$ is the capacity flow, and $N_{i}$ is the maximum possible occupancy of a cell. The CTM uses a triangular or other piecewise linear forms of the fundamental diagram, as shown in Figure 5 [39].
The traffic flow from the upstream sensor of the incident location is taken as a demand for CTM. This component of the framework, shown in Figure 1, is activated after the incident detection algorithm has detected the incident. A CTM-based approach models the incident scenario by splitting the section of the road into cells of equal lengths. Each cell $C_{n}$ between two sensors is modeled as the candidate cell.

The algorithm utilizes calibrated CTM parameters for PeMS traffic data. Dervisoglu and Gomes [40] calibrated the fundamental diagram for PeMS database at 12 different sections of freeways. Table 4 shows the calibrated traffic flow parameters that were used as input to the CTM. 

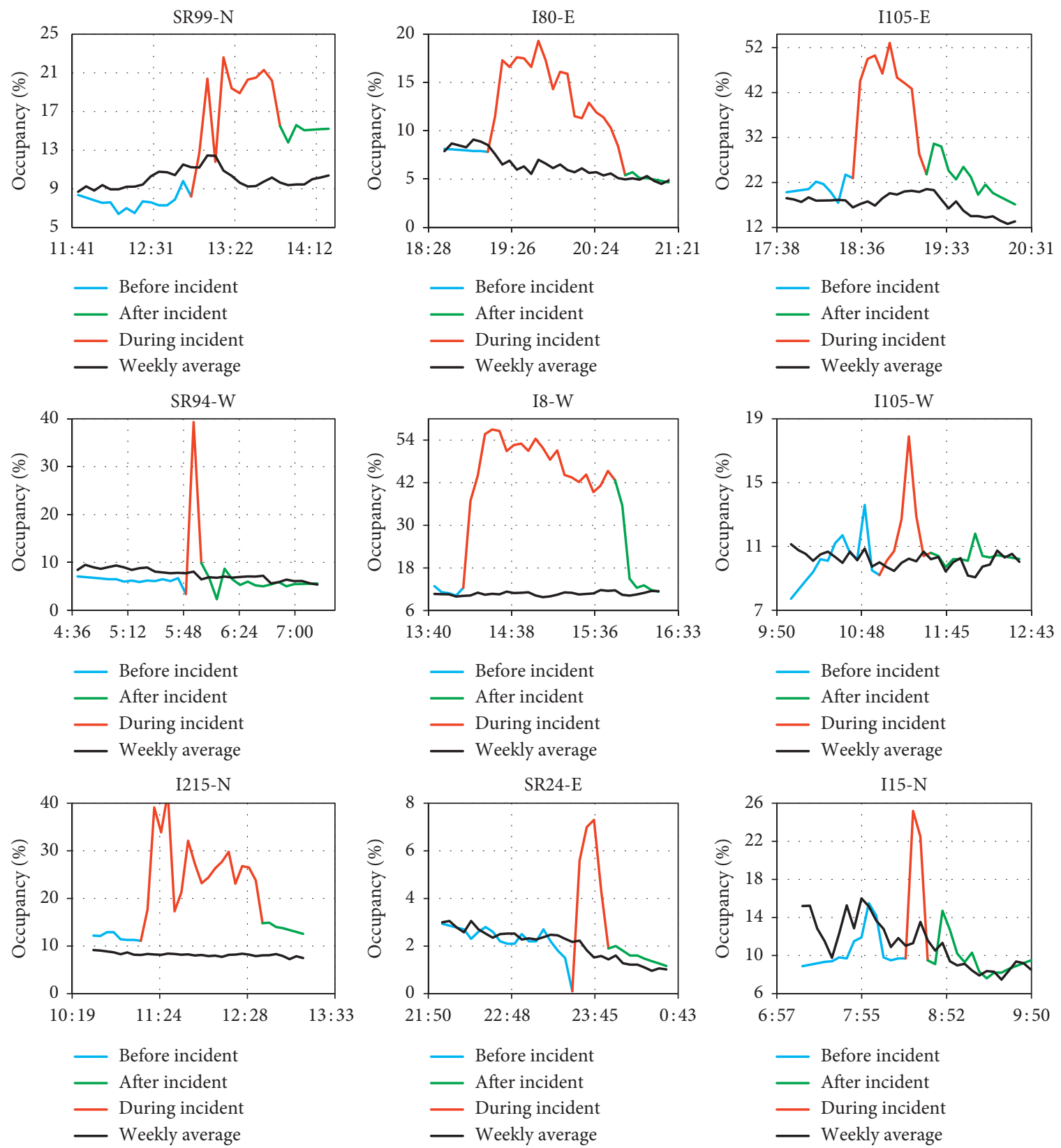

FIGURE 4: The comparison of pre-incident, during the incident and post-incident occupancies with the weekly average occupancy.

TABLE 1: Comparison of postincident speed and occupancy with the weekly average values.

\begin{tabular}{lccccc}
\hline Freeway & Day/time & $\begin{array}{c}\text { Weekly average } \\
\text { speed }(\mathrm{mph})\end{array}$ & $\begin{array}{c}\text { Reduced speed after } \\
\text { the incident }(\mathrm{mph})\end{array}$ & $\begin{array}{c}\text { Weekly average } \\
\text { occupancy }(\%)\end{array}$ & $\begin{array}{c}\text { Increased occupancy } \\
\text { after the incident }(\%)\end{array}$ \\
\hline SR99-N & $22 / 5 / 201812: 54$ & 62.63 & 36.30 & 8.82 & 20.44 \\
SR94-W & $1 / 1 / 202017: 42$ & 66.00 & 27.50 & 3.90 & 39.33 \\
I215-N & $10 / 3 / 201711: 10$ & 58.34 & 32.10 & 10.52 & 39.12 \\
I80-E & $15 / 1 / 202018: 58$ & 56.75 & 38.50 & 10.24 & 16.61 \\
I8-W & $20 / 10 / 201714: 08$ & 63.42 & 37.30 & 2.45 & 36.90 \\
SR24-E & $15 / 01 / 202023: 15$ & 70.17 & 45.30 & 20.53 & 7.0 \\
I105-E & $8 / 1 / 201818: 34$ & 56.25 & 23.50 & 4.46 & 10.62 \\
I105-W & $10 / 8 / 201811: 10$ & 62.28 & 33.90 & 11.65 & 10.73 \\
I15-N & $4 / 10 / 20188: 24$ & 68.18 & & & 25.21 \\
\hline
\end{tabular}


TABLE 2: Speed and occupancy comparison with weekly and previous time interval average.

\begin{tabular}{lcccc}
\hline Freeway & $\begin{array}{c}\text { Percentage difference of } \\
\text { upstream speed with weekly } \\
\text { average speed } \triangle \bar{U}_{k}(\%)\end{array}$ & $\begin{array}{c}\text { Percentage difference of } \\
\text { upstream speed with previous } \\
\text { time intervals } \triangle \bar{O}_{k}(\%)\end{array}$ & $\begin{array}{c}\text { Percentage difference of } \\
\text { upstream occupancy with weekly } \\
\text { average occupancy } \triangle \widehat{U}_{k}(\%)\end{array}$ & $\begin{array}{c}\text { Percentage difference of } \\
\text { upstream occupancy with } \\
\text { previous time intervals } \triangle \widehat{O}_{k} \\
(\%)\end{array}$ \\
\hline SR99-N & -42.04 & -39.74 & 56.76 & 49.84 \\
SR94-W & -58.33 & -40.80 & 79.90 & 86.26 \\
I215-N & -44.98 & -43.82 & 79.18 & 65.81 \\
I80-E & -39.03 & -26.85 & 36.63 & 47.59 \\
I8-W & -41.19 & -38.92 & 72.25 & 69.74 \\
SR24-E & -35.44 & -18.96 & 65.00 & 65.71 \\
I105-E & -58.22 & -36.08 & 16.61 & 52.04 \\
I105-W & -34.97 & -32.80 & 53.53 & 37.62 \\
I15-N & -50.28 & -43.31 & & 61.77 \\
\hline
\end{tabular}

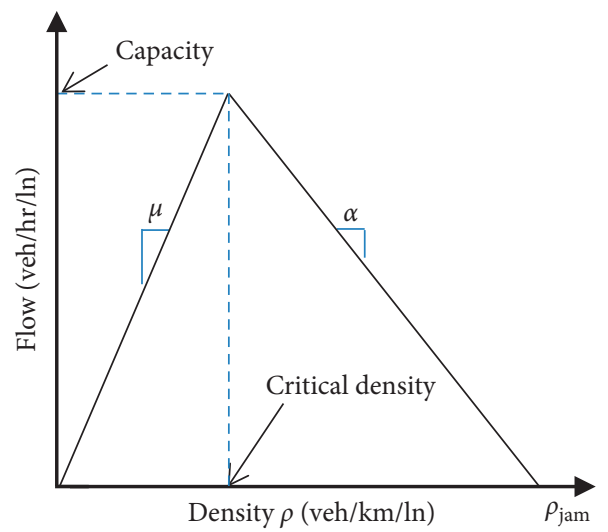

Figure 5: Fundamental Diagram used as input for CTM.

A number of scenarios are developed and simulated using calibrated CTM. The possibility of an incident occurring in each cell and its impact on each lane is modeled. The total number of scenarios is equal to the product of the number of cells and the number of lanes. Each scenario is simulated with the demand from the upstream sensor and the resulting simulated occupancy $O_{s}$ for each scenario is compared with the actual occupancy $O_{k}$. The scenario that yields the least difference in the occupancy is selected to provide the estimated location and capacity of the incident cell.

\section{Results and Discussion}

3.1. Incident Detection Using PeMS Data. The open-source data of the Department of Transportation California (PeMS) was used for the application of the model in a real environment. The incident details mentioned in Table 1 were collected from the incident log of the PeMS database. The framework proposed in Figure 1 is applied to capture the selected incidents listed in Table 1 . The results of incident detections are shown in Table 5, which compares the incident time reported by PeMS data with the time of incident detected by the algorithm.

The average difference in the reported incident time and the incident time detected by the algorithm is 9 minutes. The least difference of 1 minute was observed for the incident that occurred at I105-E. The highest difference of about 27 minutes was observed for the incident reported at I80-E on January 15, 2020, at 18:58 hrs. The incident reported on SR24-E was detected 10 minutes before its reported time. This shows the discrepancy in the incident reporting time, which must have been recorded with some error. The change in traffic flow parameters at the selected sensor of SR24-E indicates the occurrence of the incident before the reported time, which could be due to the inaccurate field report of the incident.

The observed difference in the reported incident time and the incident detection time for the selected incidents can be attributed to the following factors:

(i) Error in the reporting of the incident time.

(ii) Time taken to spill back the queue from the incident location to the upstream sensor. The incident detection algorithm is based on the change in traffic parameter values at the sensor. Therefore, this time may cause a delay in the incident detection and the accuracy of the detection time depends on the quick change in traffic conditions at the upstream sensor after the incident.

(iii) The PeMS data available and used in this study are aggregated at 5-minute intervals. The aggregation of the data for longer time intervals dilutes the impact of the incident on traffic flow parameters. 
TABLE 3: Incident detection at different intervals of percentage difference in speeds and occupancies.

\begin{tabular}{|c|c|c|c|c|c|}
\hline$\%$ Difference & Actual no. of incidents & $\Delta \bar{U}_{k}$ & $\begin{array}{l}\triangle \bar{O}_{k} \\
\text { No. }\end{array}$ & $\begin{array}{l}\Delta \widehat{U}_{k} \\
\text { ected }\end{array}$ & $\Delta \widehat{O}_{k}$ \\
\hline 10 & 9 & 9 & 9 & 9 & 9 \\
\hline 20 & 9 & 9 & 8 & 7 & 9 \\
\hline 30 & 9 & 9 & 7 & 7 & 9 \\
\hline 40 & 9 & 6 & 3 & 6 & 8 \\
\hline 50 & 9 & 3 & 0 & 6 & 6 \\
\hline 60 & 9 & 0 & 0 & 4 & 5 \\
\hline 70 & 9 & 0 & 0 & 3 & 1 \\
\hline 80 & 9 & 0 & 0 & 0 & 1 \\
\hline 90 & 9 & 0 & 0 & 0 & 0 \\
\hline
\end{tabular}

TABLE 4: PEMS calibrated fundamental diagram parameters $[40,41]$.

\begin{tabular}{lc}
\hline Traffic flow parameters & Value \\
\hline Free flow speed (mph) & 65 \\
Capacity (veh/hr/lane) & 1861 \\
Jam density (veh/mile/lane) & 168 \\
Critical density (veh/mile/lane) & 29 \\
Shockwave speed (mph) & 14 \\
\hline
\end{tabular}

TABLE 5: Comparison of incident time and detection time.

\begin{tabular}{lcc}
\hline Freeway & Reported incident time & Detection time \\
\hline SR99-N & $12: 54$ & $13: 00$ \\
SR94-W & $17: 42$ & $17: 55$ \\
I215-N & $11: 10$ & $11: 20$ \\
I80-E & $18: 58$ & $19: 25$ \\
I8-W & $14: 08$ & $14: 10$ \\
SR24-E & $23: 15$ & $23: 05$ \\
I105-E & $18: 34$ & $18: 35$ \\
I105-W & $11: 10$ & $11: 20$ \\
I15-N & $8: 24$ & $8: 30$ \\
\hline
\end{tabular}

3.2. Capacity Estimation. When an incident is detected in the system, the component of the framework for capacity estimation is activated. The methodology of the capacity estimation algorithm is discussed in Section 2.2. Figure 6 shows the results of the capacity estimation of all nine incidents. The capacity profile is compared with the speed data to highlight the variation in traffic conditions with the change in capacity.

Figure 6 illustrates the estimated capacity profiles at the selected freeway sections. The estimated capacity profile is compared with the speed profile to highlight the performance of the proposed incident algorithm.

Table 6 shows the estimated capacity as a function of the available number of lanes for all the incidents selected in this study. In PeMS data, the number of lanes affected due to the incident is not included in the incident report. Therefore, the capacity flow or the number of affected lanes due to the incident is not known. The accuracy of estimated capacity in the absence of actual value of reduced capacity cannot be measured. However, the performance of the proposed algorithm can be observed in
Figure 6, which shows that the estimated capacity changes with the change in traffic conditions measured at the sensor.

3.3. Incident Location Estimation. This study estimates the location of the incident in real time as well. The location and capacity during the incident are estimated simultaneously, using the framework described in Figure 1. The scenario, for which the difference between simulated occupancy and measured occupancy is the least, is selected as the outcome for estimated capacity and location. Figures 7-9 show the comparison of actual occupancy with the simulated occupancy.

In Figure 7, the increase in occupancy is observed at 5:55 PM at the selected sensor of freeway SR94-W after the incident at its downstream. According to the incident report, the incident occurred at 5:42 PM, and the lag in occupancy increase is due to the time required for queue propagation to the upstream senor. The actual occupancy is restored to normal condition in the next interval at $6: 00$ PM, while the simulated occupancy restored to normal conditions with a lag of one time interval ( 5 minutes). The incident occurred at $576 \mathrm{~m}$ from the sensor. The algorithm estimated the location of the incident in the second cell. The second cell starts at $300 \mathrm{~m}$ and ends at $600 \mathrm{~m}$. Thus, the proposed algorithm accurately estimated the location of this incident.

Figure 8 illustrates the occupancy profile of the freeway I105-E when an incident is detected in the second cell. The occupancy increases after the incident at 18:35. According to the incident details mentioned in Table 1 , the incident was reported at 18:34. The increased occupancy at the sensor was observed at $18: 35$. The simulated occupancy shows a similar trend to that of the actual occupancy, which indicates that the second cell is the incident cell. Furthermore, the actual location of the incident is also estimated accurately in the second cell, which is $320 \mathrm{~m}$ away from the upstream sensor.

The occupancy profile of freeway I105-W is shown in Figure 9. The increase in occupancy can be seen in two places. The increased occupancy at 10:50 hrs was found to be a daily variation. The second peak in the occupancy profile is due to the incident because the reduction in speed is also observed at the same time in Figure 3. The time of the incident mentioned in Table 1 also verifies the increase in 

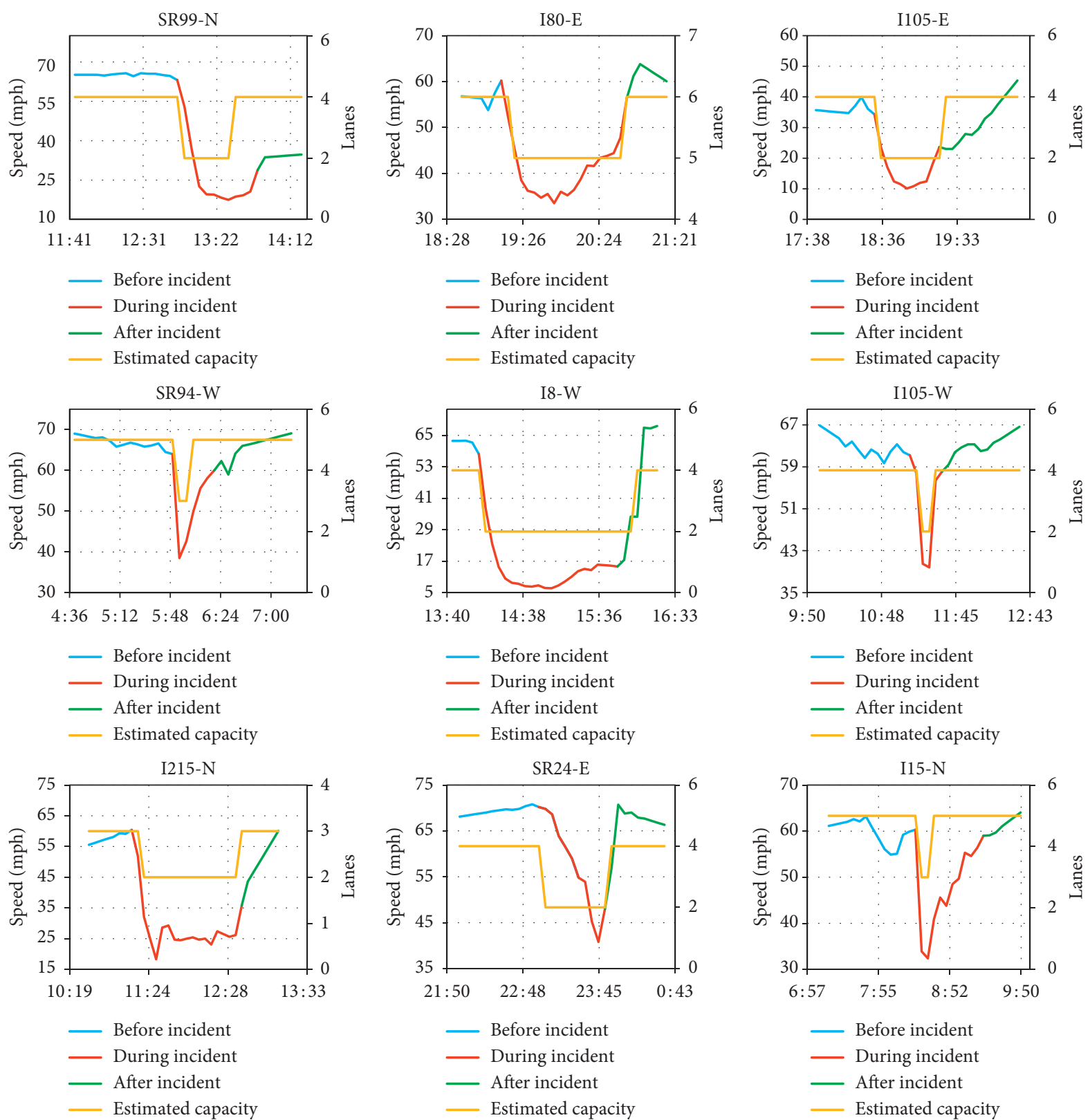

FIgURE 6: Comparison of estimated capacities with the speed profile.

TABLE 6: Details of the estimated capacity during the incident as a function of available lanes.

\begin{tabular}{lcc}
\hline Freeway & $\begin{array}{c}\text { Capacity available } \\
\text { (total number of lanes) }\end{array}$ & Estimated reduced capacity \\
\hline SR99-N & 4 & 2 \\
SR94-W & 5 & 2 \\
I215-N & 3 & 2 \\
I80-E & 6 & 5 \\
I8-W & 4 & 2 \\
SR24-E & 4 & 2 \\
I105-E & 4 & 2 \\
I105-W & 4 & 2 \\
I15-N & 5 & 3 \\
\hline
\end{tabular}

occupancy. The incident occurred at $480 \mathrm{~m}$ from the upstream sensor, which corresponds to the second cell. The estimated location of the incident is also the second cell, which shows the accurate performance of the proposed algorithm.

Table 7 shows the estimated locations of all nine incidents. Locations of six out of nine incidents were estimated accurately with an overall accuracy of $92.5 \%$

The sections of the freeways affected with the incident are shown in Figure 10. In each section, the distance between the upstream and downstream sensors was divided into cells of equal length of $300 \mathrm{~m}$. The shaded cell represents the estimated incident cell, in which the yellow strip indicates 


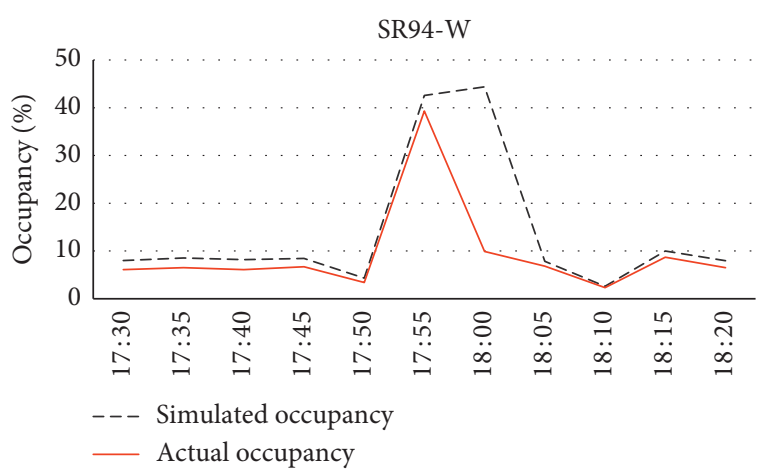

FIGURE 7: Actual and simulated occupancy comparison at freeway SR94-W.

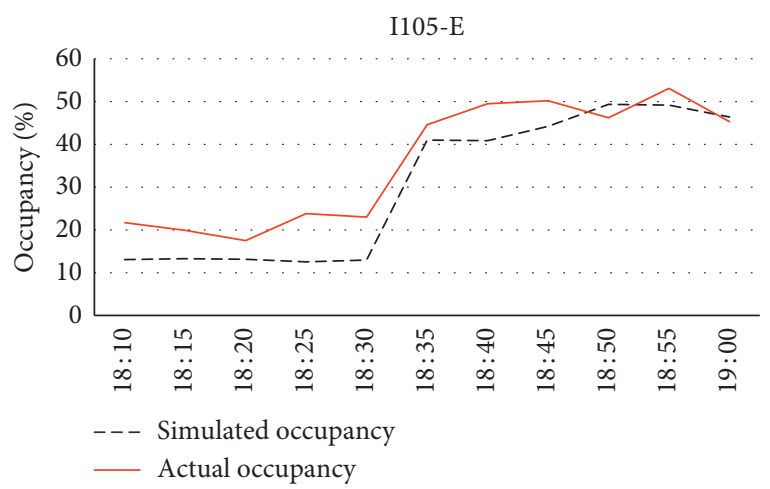

FIgURE 8: Actual and simulated occupancy comparison at freeway I105-E.

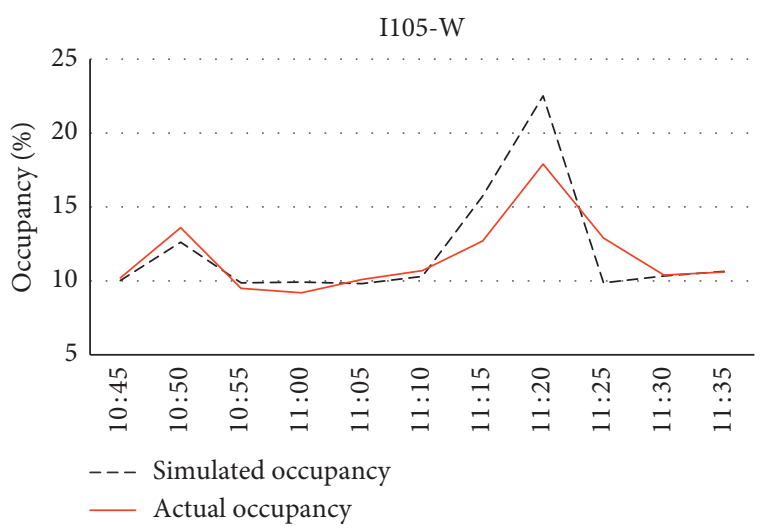

Figure 9: Actual and simulated occupancy comparison at freeway I105-W.

the location of the upstream sensor, while the red strip shows the location of the downstream sensor. The pin location icon in red color shows the actual location of the incident from the PeMS data.

3.4. Application of Framework in a Simulated Environment. The outcomes of the model implementation on field data are shown in previous sections, showing the acceptable performance of the proposed algorithm. However, there is a slight deviation when the model outputs are compared with the field data. There are two main reasons for this error in the incident detection and capacity estimation:

(i) There are off-ramps and on-ramps between the selected sensors. The propagation of the impact of the incident on capacity and other traffic flow parameters to the upstream sensor can be significantly affected if there is an on-ramp or off-ramp between the two sensors. For example, if there is an off-ramp between the incident location and the upstream sensor, then the vehicles exiting the road section could dilute the impact of the incident on the upstream sensor and may cause some error in the estimation. The proposed model could yield more accurate results if there are no on-ramps or offramps between the sensors. This deficiency may also be improved by integrating the measurements obtained from the on-ramp and off-ramp sensors in the incident detection and estimation algorithm.

(ii) The inherent systematic error in measuring the traffic flow and error in recording the details of the incident are another factor that makes it difficult to measure the accuracy of the proposed framework. For example, due to the error in the incident reporting time, it becomes difficult to estimate the accuracy of the incident detection algorithm.

Due to the factors mentioned above, the proposed framework was applied to a simulated environment. In the simulated scenario, all the information about an incident, such as incident occurring time, location, duration, and capacity reduction, is known.

The proposed framework was applied to a $5 \mathrm{~km}$ long road section, having 4 lanes, as shown in Figure 11. The section is a mid-block section with no on-ramp and off-ramp. It is divided into 10 cells of equal lengths of $500 \mathrm{~m}$. The free-flow speed is taken at $60 \mathrm{~km} / \mathrm{hr}$. There are loop detectors at the beginning and the end of the road segment. The sensors provide measurements at 30-second intervals. The incident occurred in cell 2 at $750 \mathrm{~m}$ from the upstream detector for 10 minutes from time-step 30 to 50 , which decreased the capacity of the road section to 2 lanes from 4 lanes.

The incident detection algorithm requires weekly average values of measured speed and occupancy at the same time interval. For this purpose, synthetic measurements were generated by assuming traffic demand around $7000 \mathrm{veh} / \mathrm{hr}$ with a slight variation on each day. The outcomes of the model application are shown in Figure 12, which shows that the proposed algorithm accurately detected the incident and estimated the reduced capacity. The incident occurred at the 30th time-step, which was detected by the algorithm at time-step 33, showing a lag of 1.5 minutes. The incident was cleared at the 50th time-step, which was estimated at time-step 52 . Figure 12 shows the variation in capacity profile. Similarly, the proposed algorithm accurately estimated the reduced capacity due to the incident. Furthermore, the estimated location of the incident was also estimated accurately at cell 2 . 
TABLE 7: Details of location estimation of incident.

\begin{tabular}{lcccc}
\hline Freeway & Actual incident location from upstream detector & Estimated location & Accuracy (\%) & Overall accuracy (\%) \\
\hline SR99-N & Cell 1 & Cell 1 & 100 & \\
SR94-W & Cell 1 & Cell 1 & 100 & \\
I215-N & Cell 2 & Cell 2 & 100 & \\
I80-E & Cell 2 & Cell 1 & 67 & 979 \\
I8-W & Cell 2 & Cell 3 & 79 & 92.5 \\
SR24-E & Cell 2 & Cell 2 & 100 & \\
I105-E & Cell 2 & Cell 2 & 100 & \\
I105-W & Cell 2 & Cell 2 & 100 & \\
I15-N & Cell 2 & Cell 1 & 87 & \\
\hline
\end{tabular}

SR99-N

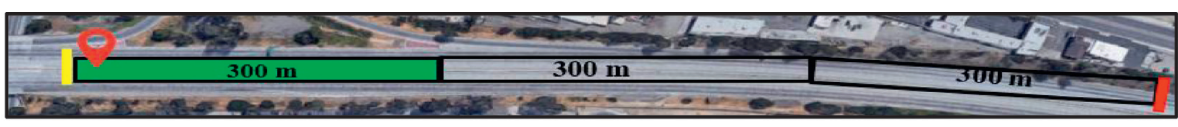

SR94-W

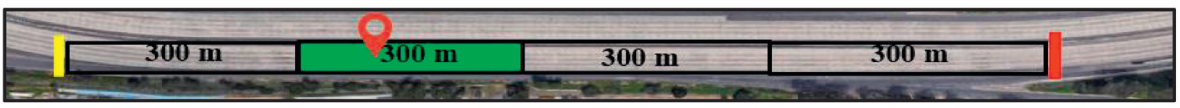

I215-N

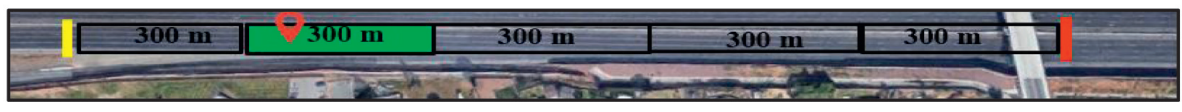

I80-E

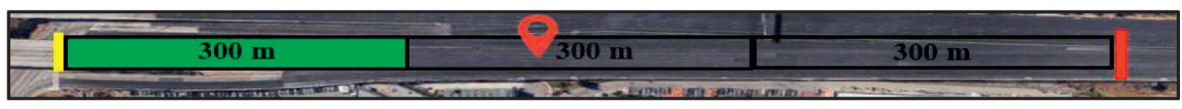

I8-W

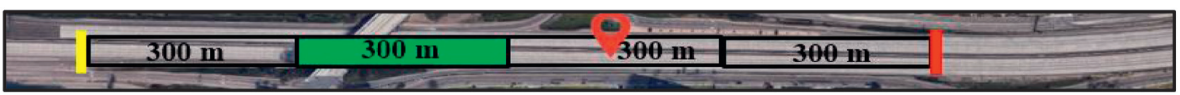

SR24-E

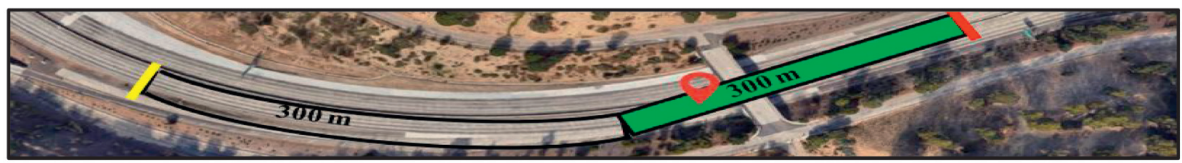

I105-E

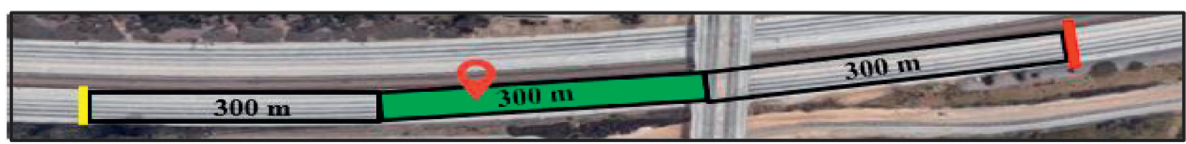

I105-W

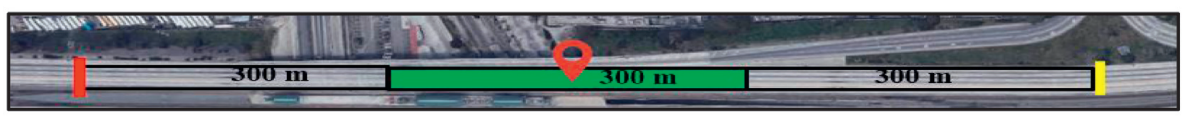

I15-N

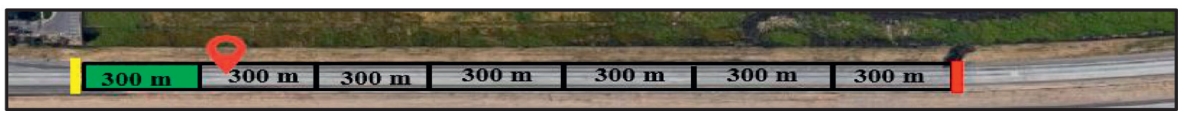

$\begin{array}{lll}\text { Location of upstream sensor } & \text { Incident cell } \\ \text { Actual location of incident } & \text { Location of downstream sensor }\end{array}$

Figure 10: Comparison of the estimated and actual incident locations. 


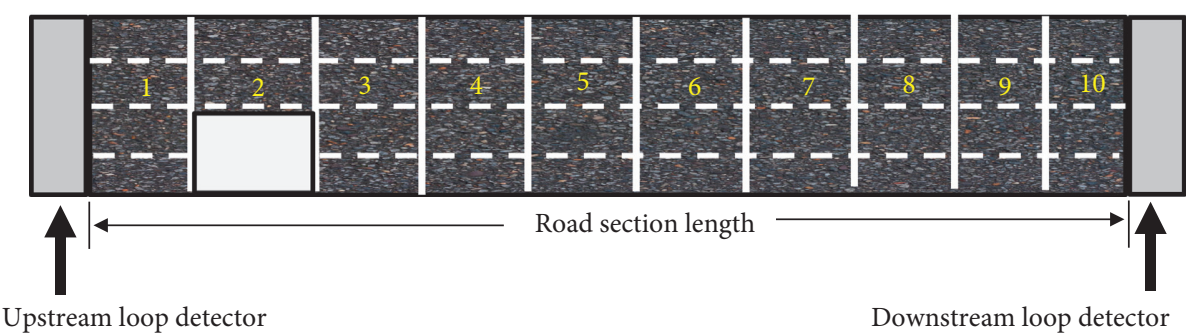

FIgUre 11: Model setup for simulated reality.

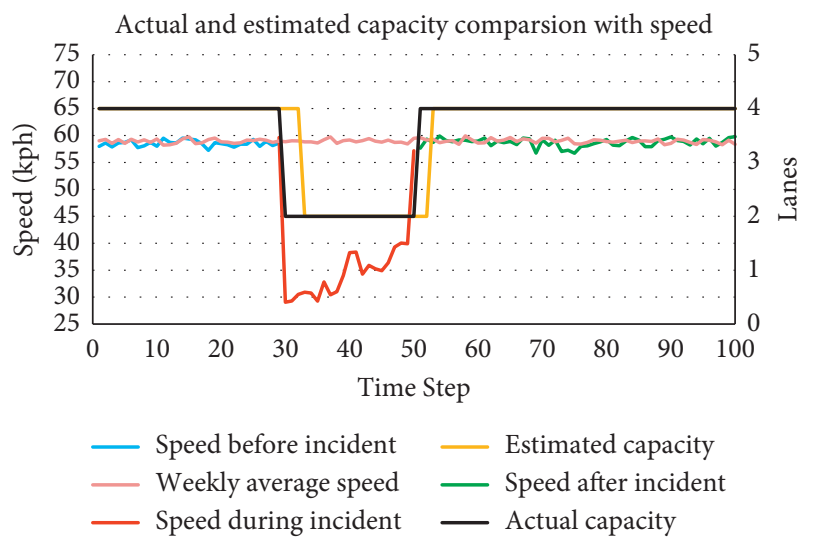

FIGURE 12: Comparison of estimated capacity with actual capacity.

\section{Conclusions and Recommendations}

This study proposed a framework that utilizes the measurements obtained from the inductance loop detector for incident detection, estimation of reduced capacity, and estimation of the location of the incident as well. The proposed model is developed using the sensor data available from PeMS and could be applied in real time without any modifications. The proposed algorithm was able to accurately detect the incident and estimate the reduced capacity of the road section and the location of the incident. The location of the incident is estimated with an overall accuracy of $92.5 \%$. The following conclusions are drawn from this study:

The proposed algorithm requires the measurements of macroscopic parameters such as flow, speed, and occupancy. The utilization of macroscopic traffic flow parameters and limited macroscopic data makes the proposed algorithm computationally more efficient, which makes it more suitable for real-time applications.

The proposed algorithm is applicable when the effect of the incident is observed at the sensor located upstream of the incident location. The minor incidents, when traffic demand is low and no congestion is caused due to the incident, cannot be detected by the algorithm. However, the incidents not causing a significant impact on traffic conditions do not need real-time traffic control and incident management measures, which are otherwise needed.

The incident is detected when both speed and occupancy exceed the threshold limits. Incidents from PeMS database were identified to develop the threshold limits for speed and occupancy. The threshold limits are based on the average values of speed and occupancy from the recorded observations.

The distance between the incident location and the upstream sensor affects the accuracy of the incident detection algorithm. The time lag of detection may increase with the increase in the distance between the incident location and the sensor, as the queue built-up and spillback time increase with this distance. The traffic condition may also affect this time lag.

It was observed from the data that the incident reporting time and duration of the incidents reported in PeMS database do not match with the observed data. This deviation also affects the estimation of the accuracy of the proposed algorithm.

The incident detection and capacity estimation algorithm was applied to the nine selected incidents to evaluate its performance. The location of the incident was estimated with an average accuracy of $92.5 \%$. The average difference between incident reporting and detection time was 9.8 minutes, which is less than two time intervals, as the PeMS data are reported at 5 -minute intervals. The accuracy of the number of affected lanes cannot be determined as the PeMS data do not provide this information in the incident report.

The accuracy of the incident detection of the proposed algorithm can be further improved when the traffic flow parameters are provided with higher resolution. The aggregation of the data over 5-minute interval dilutes the impact of the incident, especially during the interval when the incident occurred. In reality, the inductance loop detectors provide measurements at 30-second interval. The use of measurements at 30-second interval is expected to improve the estimation accuracy.

The proposed framework was also applied to a simulated controlled environment. The model detected the incident and estimated the capacity and location of the incident accurately.

The information of capacity variation is beneficial for the estimation of traffic state. The accurate detection of the incident and capacity estimation are crucial in real-time traffic and incident management. The proposed algorithm enables the traffic controller to devise a strategy that effectively manages congestion and improves network 
performance during the incident. Effective incident management reduces the time spent by drivers in congestion, which may result in less anxiety and stress, thus contributing to improving the road safety of commuters.

The performance of the proposed framework may be improved by using CTM-based estimation techniques such as CTM-KF (Kalman Filter) or CTM-EKF (Extended Kalman Filter). Furthermore, data from other available sources (Bluetooth, Wi-Fi, GSM, and GPS-based sensors) could be integrated to develop improved algorithms. The autonomous and connected vehicles could provide more useful and accurate information for incident detection and capacity estimation. With a reasonable penetration of autonomous and connected vehicles, the real-time information from these vehicles could be fused with sensor data to develop more efficient algorithms.

\section{Data Availability}

The data used in this research are publicly available and can be downloaded from the PeMS website (http://pems.dot.ca. gov/).

\section{Conflicts of Interest}

The authors declare that there are no conflicts of interest.

\section{Authors' Contributions}

Yongjun Shen was responsible for funding acquisition, resources, supervision, and writing review and editing; Syed Muzammil Abbas provided the software; Afzal Ahmed performed validation; Syed Muzammil Abbas and Afzal Ahmed contributed in the methodology and writing original draft.

\section{Acknowledgments}

This research was supported by the National Key Research and Development Project (Grant no. 2018YFE0102700) and the National Natural Science Foundation of China (Grant no. 71701045). Dr. Afzal Ahmed's contribution to this research was supported by Exascale Open Data Analytics Lab, National Center for Big Data and Cloud Computing (funded by Higher Education Commission, Pakistan).

\section{References}

[1] N. Owens, Traffic Incident Management Handbook, Federal Highway Administration, Washington, U.S, 2010.

[2] T. F. Golob, W. W. Recker, and V. M. Alvarez, "Freeway safety as a function of traffic flow," Accident Analysis \& Prevention, vol. 36, no. 6, pp. 933-946, 2004.

[3] J. Sun, T. Li, F. Li, and F. Chen, "Analysis of safety factors for urban expressways considering the effect of congestion in Shanghai, China," Accident Analysis \& Prevention, vol. 95, pp. 503-511, 2016.

[4] L.-G. Mattsson and E. Jenelius, "Vulnerability and resilience of transport systems-a discussion of recent research," Transportation Research Part A: Policy and Practice, vol. 81, pp. 16-34, 2015.
[5] M. A. Goldberg, "On the inefficiency of being efficient," Environment and Planning A: Economy and Space, vol. 7, no. 8, pp. 921-939, 1975.

[6] V. L. Knoop, S. P. Hoogendoorn, and H. J. Van Zuylen, "Capacity reduction at incidents," Transportation Research Record: Journal of the Transportation Research Board, vol. 2071, no. 1, pp. 19-25, 2008.

[7] J. Kwon, M. Mauch, and P. Varaiya, "Components of congestion," Transportation Research Record: Journal of the Transportation Research Board, vol. 1959, no. 1, pp. 84-91, 2006.

[8] F. Ahmed and Y. E. Hawas, "A threshold-based real-time incident detection system for urban traffic networks," Procedia-Social and Behavioral Sciences, vol. 48, pp. 1713-1722, 2012.

[9] J.-B. Sheu, "A sequential detection approach to real-time freeway incident detection and characterization," European Journal of Operational Research, vol. 157, no. 2, pp. 471-485, 2004.

[10] R. Rossi, M. Gastaldi, G. Gecchele, and V. Barbaro, "Fuzzy logic-based incident detection system using loop detectors data," Transportation Research Procedia, vol. 10, pp. 266-275, 2015.

[11] Z. Zhang, Q. He, J. Gao, and M. Ni, "A deep learning approach for detecting traffic accidents from social media data," Transportation Research Part C: Emerging Technologies, vol. 86, pp. 580-596, 2018.

[12] Y. Gu, Z. Qian, and F. Chen, "From Twitter to detector: realtime traffic incident detection using social media data," Transportation Research Part C: Emerging Technologies, vol. 67, pp. 321-342, 2016.

[13] J. Xiao, "SVM and KNN ensemble learning for traffic incident detection," Physica A: Statistical Mechanics and Its Applications, vol. 517, pp. 29-35, 2019.

[14] A. Kinoshita, A. Takasu, and J. Adachi, "Real-time traffic incident detection using a probabilistic topic model," Information Systems, vol. 54, pp. 169-188, 2015.

[15] C. Oh, "Real-time estimation of freeway accident likelihood," in Proceedings of the 80th Annual Meeting of the Transportation Research Board, Washington, DC, USA, November 2001.

[16] F. Chen, S. Chen, and X. Ma, "Analysis of hourly crash likelihood using unbalanced panel data mixed logit model and real-time driving environmental big data," Journal of Safety Research, vol. 65, pp. 153-159, 2018.

[17] Y. Wang and M. Papageorgiou, "Real-time freeway traffic state estimation based on extended kalman filter: a general approach," Transportation Research Part B: Methodological, vol. 39, no. 2, pp. 141-167, 2005.

[18] D. Ngoduy, "Kernel smoothing method applicable to the dynamic calibration of traffic flow models," Computer-Aided Civil and Infrastructure Engineering, vol. 26, no. 6, pp. 420432, 2011.

[19] A. Ahmed, Integration of Real-Time Traffic State Estimation and Dynamic Traffic Assignment with Applications to Advanced Traveller Information Systems, University of Leeds, Leeds, England, 2015.

[20] A. Ahmed, D. Ngoduy, and D. Watling, "Prediction of traveller information and route choice based on real-time estimated traffic state," Transportmetrica B: Transport Dynamics, vol. 4, no. 1, pp. 23-47, 2016.

[21] A. Ahmed, S. A. A. Naqvi, D. Watling, and D. Ngoduy, "Realtime dynamic traffic control based on traffic-state estimation," 
Transportation Research Record: Journal of the Transportation Research Board, vol. 2673, no. 5, pp. 584-595, 2019.

[22] F. Chen, S. Chen, and X. Ma, "Crash frequency modeling using real-time environmental and traffic data and unbalanced panel data models," International Journal of Environmental Research and Public Health, vol. 13, no. 6, p. 609, 2016.

[23] B. L. Smith, L. Qin, and R. Venkatanarayana, "Characterization of freeway capacity reduction resulting from traffic accidents," Journal of Transportation Engineering, vol. 129, no. 4, pp. 362-368, 2003.

[24] M. Hadi, P. Sinha, and A. Wang, "Modeling reductions in freeway capacity due to incidents in microscopic simulation models," Transportation Research Record: Journal of the Transportation Research Board, vol. 1999, no. 1, pp. 62-68, 2007.

[25] C. Wang, M. Quddus, and S. Ison, “A spatio-temporal analysis of the impact of congestion on traffic safety on major roads in the UK," Transportmetrica A: Transport Science, vol. 9, no. 2, pp. 124-148, 2013.

[26] A. Almotahari, "Analysis of incident-induced capacity reductions for improved delay estimation," Journal of Transportation Engineering, Part A: Systems, vol. 145, no. 2, Article ID 04018083, 2019.

[27] R. L. Bertini and A. M. Myton, "Use of performance measurement system data to diagnose freeway bottleneck locations empirically in orange county, California," Transportation Research Record: Journal of the Transportation Research Board, vol. 1925, no. 1, pp. 48-57, 2005.

[28] K. Nie, Z. Wang, Q. Du, F. Ren, and Q. Tian, “A networkconstrained integrated method for detecting spatial cluster and risk location of traffic crash: a case study from wuhan, China," Sustainability, vol. 7, no. 3, pp. 2662-2677, 2015.

[29] M. A. Quddus, "Modelling area-wide count outcomes with spatial correlation and heterogeneity: an analysis of London crash data," Accident Analysis \& Prevention, vol. 40, no. 4, pp. 1486-1497, 2008.

[30] A. J. Khattak, X. Wang, and H. Zhang, "Spatial analysis and modeling of traffic incidents for proactive incident management and strategic planning," Transportation Research Record: Journal of the Transportation Research Board, vol. 2178, no. 1, pp. 128-137, 2010.

[31] W. Cheng and S. Washington, "New criteria for evaluating methods of identifying hot spots," Transportation Research Record: Journal of the Transportation Research Board, vol. 2083, no. 1, pp. 76-85, 2008.

[32] V. Prasannakumar, H. Vijith, R. Charutha, and N. Geetha, "Spatio-temporal clustering of road accidents: GIS based analysis and assessment," Procedia-Social and Behavioral Sciences, vol. 21, pp. 317-325, 2011.

[33] T. Steenberghen, T. Dufays, I. Thomas, and B. Flahaut, "Intraurban location and clustering of road accidents using GIS: a Belgian example," International Journal of Geographical Information Science, vol. 18, no. 2, pp. 169-181, 2004.

[34] M. Bíl, R. Andrášik, and Z. Janoška, "Identification of hazardous road locations of traffic accidents by means of kernel density estimation and cluster significance evaluation," Accident Analysis \& Prevention, vol. 55, pp. 265-273, 2013.

[35] T. K. Anderson, "Kernel density estimation and K-means clustering to profile road accident hotspots," Accident Analysis \& Prevention, vol. 41, no. 3, pp. 359-364, 2009.

[36] A. Ahmed, D. Watling, and D. Ngoduy, "Significance of sensor location in real-time traffic state estimation," Procedia Engineering, vol. 77, pp. 114-122, 2014.
[37] J. Pan, I. S. Popa, K. Zeitouni, and C. Borcea, "Proactive vehicular traffic rerouting for lower travel time," IEEE Transactions on Vehicular Technology, vol. 62, no. 8, pp. 3551-3568, 2013.

[38] C. F. Daganzo, "The cell transmission model: a dynamic representation of highway traffic consistent with the hydrodynamic theory," Transportation Research Part B: Methodological, vol. 28, no. 4, pp. 269-287, 1994.

[39] A. Ahmed, "Width-based cell transmission model for heterogeneous and undisciplined traffic streams," Transportation Research Record, vol. 2673, no. 5, pp. 682-692, 2019.

[40] G. Dervisoglu, "Automatic calibration of the fundamental diagram and empirical observations on capacity," in Proceedings of the Transportation Research Board 88th Annual Meeting, Washington, DC, USA, January 2009.

[41] Safemotorist.Com. California Speed Limits 2020 https://www. safemotorist.com/California/Roads/speed/. 